\title{
De Finetti Theorems for Braided Parafermions
}

\author{
Kaifeng Bu ${ }^{1,2}$, Arthur Jaffe ${ }^{1}$, Zhengwei Liu ${ }^{3,1}$, Jinsong $\mathbf{W u}^{4,1}$ \\ ${ }^{1}$ Harvard University, Cambridge, MA 02138, USA. E-mail: kfbu@ fas.harvard.edu; jaffe@ g.harvard.edu \\ 2 Zhejiang University, Hangzhou, China \\ 3 Tsinghua University, Beijing, China. E-mail: liuzhengwei@mail.tsinghua.edu.cn \\ ${ }^{4}$ Harbin Institute of Technology, Harbin, China. E-mail: wjs@hit.edu.cn
}

Received: 9 June 2018 / Accepted: 29 July 2019

Published online: 25 October 2019 - (c) The Author(s) 2019

\begin{abstract}
The classical de Finetti theorem in probability theory relates symmetry under the permutation group with the independence of random variables. This result has application in quantum information. Here we study states that are invariant with respect to a natural action of the braid group, and we emphasize the pictorial formulation and interpretation of our results. We prove a new type of de Finetti theorem for the fourstring, double-braid group acting on the parafermion algebra to braid qudits, a natural symmetry in the quon language for quantum information. We prove that a braid-invariant state is extremal if and only if it is a product state. Furthermore, we provide an explicit characterization of braid-invariant states on the parafermion algebra, including finding a distinction that depends on whether the order of the parafermion algebra is square free. We characterize the extremal nature of product states (an inverse de Finetti theorem).
\end{abstract}

\section{Contents}

1. Introduction . . . . . . . . . . . . . . . . . 435

2. Parafermion Algebras and the PAPPA Model . . . . . . . . . . . . . . . 437

3. The Four-String Braid Group $\mathbb{B}_{\infty} \ldots \ldots$. . . . . . . . . . . . . . . . 438

4. The Tail Algebra for Parafermions . . . . . . . . . . . . . . . . . . . . . . . . . . 442

5. The Inverse de Finetti Theorem . . . . . . . . . . . . . . . . . . . . . . . 447

6. The de Finetti Theorem: Square-Free $d$, the Neutral Case . . . . . . . . . 449

7. The de Finetti Theorem: The Non-neutral Case . . . . . . . . . . . . . . . 451

8. Summary ....................... . . . 454

Acknowledgements . . . . . . . . . . . . . . . . . 454

References ............................. 454

\section{Introduction}

1.1. Background. The famous de Finetti theorem in classical probability theory clarifies the relationship between permutation symmetry and the independence of a sequence of 
random variables [dF31, dF37,EL55]. Consequently an infinite sequence of symmetric random variables can be written as a convex combination of an independent identically distributed (i.i.d.) sequence.

Størmer [Sto69] proposed a non-commutative (quantum) version of the de Finetti theorem, and he demonstrated that extremal, symmetric states on infinite, tensor-product $C^{*}$ algebras can be expressed in terms of product states. Other symmetry groups yield noncommutative formulations of de Finetti theorems, and braid invariance has been considered by Gohm and Köstler in [GK09, K10]. The de Finetti theorem has been extended to noncommutative probability theory, with a classical probability measure being replaced by quantum state [KS09, Cur09, Cur10, GK10, CF12, BCS12, DK14,Liu15,Liu17].

Diaconis and Freedman established a de Finetti theorem for a finite (rather than infinite) sequence of exchangeable random variables [DF80]. This led to various types of de Finetti theorems in statistical physics and in quantum information [HM,FLV88, RW89,CFS02, KR05, Ren07,CK07,BL09,CT09]. König and Renner [KR05] showed that any $k$-partite reduced state arising from a state on $n$ systems that is permutationsymmetric, with $k \ll n$, is close to a convex combination of i.i.d. n-partite states. Here i.i.d. means that the state $\varphi=\rho^{\otimes n}$ can be written as a product of identical copies.

This result is crucial for understanding the structure of permutation-symmetric states, and especially for the consideration of quantum entanglement of such states [HHHH09]. The use of such states has application in quantum information processing tasks ranging from entanglement testing [BrCY11], quantum key distribution [Ren05], quantum hypothesis testing [BrP10], to quantum state tomography [Ren07], and quantum complexity theory [BrCY11,LS15, BrH17].

Non-abelian statistics of quasiparticle models allow one to perform topological quantum computation, such as in the zero-mode model for Majorana fermions (the $d=2$ case of parafermions) [Kit03,NSS+08]. Parafermions, as a generalization of Majoranas, have recently attracted much attention in condensed matter physics [Fen12,LBRS12, YW12] and [CAS13, MCA+14, KL14,HWL15]. We have given a natural, pictorial representation of the parafermion algebra and showed how this yields a pictorial representation of their Clifford gates [JLW18,JLW17,LWJ17].

1.2. New results. Here we present a de Finetti theorem for states on parafermion algebras of order $d$. In particular, we use the fact that a pair of parafermions of order $d$ generate the $d \times d$ matrix algebra $\mathbb{M}_{d}(\mathbb{C})$, that we denote by $P F_{2}$. Thus it is natural to consider pairs of parafermions as a unit, and to study double braids that exchange these pairs.

The infinite parafermion algebra $P F_{\infty}$ is a $\mathbb{Z}_{d}$-graded, tensor product of algebras $P F_{2}$ of parafermion pairs. Here we consider the braid group $\mathbb{B}_{\infty}$, as defined in Sect. 3.1, acting on pairs of parafermions . Let $S_{\mathbb{B}_{\infty}}$ denote the states on $P F_{\infty}$ that are invariant under the action of $\mathbb{B}_{\infty}$. In Sects. 6-7 we prove:

Theorem 1 (de Finitti for braided parafermions). Let $\varphi \in S_{\mathbb{B}_{\infty}}$ be a braid-invariant state on $P F_{\infty}$. Then the following are equivalent:

(1) The state $\varphi$ is extremal in the set of states $S_{\mathbb{B}_{\infty}}$ on $P F_{\infty}$.

(2) The state $\varphi=\rho^{\otimes \infty}$ is the infinite tensor product of a state $\rho$ on $P F_{2}$.

We can refine this characterization, depending on the order $d$ of the algebra $P F_{\infty}$. This results in different restrictions on $\rho$. As a consequence of Theorem 1 , any $\mathbb{B}_{\infty^{-}}$ invariant state on $P F_{\infty}$ is in the closure of the convex hull of the product states. Let $\overline{P F}_{\infty}^{\varphi}$ denote the von Neumann algebra generated by $P F_{\infty}$ in the Gelfand-NaimarkSegal (GNS) construction with respect to the state $\varphi \in S_{\mathbb{B}_{\infty}}$. Also let $\left(\overline{P F}_{\infty}^{\varphi}\right)^{\mathbb{B}_{\infty}}$ be the 
fixed point algebra under the action of the braid group $\mathbb{B}_{\infty}$. The neutral subalgebra of $\left(\overline{P F}_{\infty}^{\varphi}\right)^{\mathbb{B}_{\infty}}$ is the subalgebra generated by monomials in parafermions of degree zero $\bmod d$.

It is interesting that a distinction arises in this characterization, according to whether or not the order of the parafermion algebra is square free. (This means that $d=\prod_{i} p_{i}$, where the primes $p_{i}$ are distinct.) Let us now suppose that the degree $d$ of the parafermion algebra is square free. In this case one finds that extremal, braid-invariant states are neutral and that they give rise to a factor. One can refine Theorem 1 as follows:

Theorem 2. Let $\varphi \in S_{\mathbb{B}_{\infty}}$ be a braid-invariant state on a parafermion algebra $P F_{\infty}$ of square-free degree $d$. The following are equivalent:

(1) The state $\varphi$ is extremal in $S_{\mathbb{B}_{\infty}}$.

(2) The state $\varphi=\rho^{\otimes \infty}$, where $\rho$ is a neutral state on $P F_{2}$.

(3) The neutral subalgebra of $\left(\overline{P F}_{\infty}^{\varphi}\right)^{\mathbb{B}_{\infty}}=\mathbb{C}$.

(4) The algebra $\left(\overline{P F}_{\infty}^{\varphi}\right)^{\mathbb{B}_{\infty}}=\mathbb{C}$.

(5) The von Neumann algebra $\overline{P F}_{\infty}^{\varphi}$ is a factor.

In case $d$ is not square free, we give the corresponding characterization braid-invariant states and their von Neumann algebras in Theorems 21 and 22. As this requires some additional terminology that we only introduce later, we postpone these statements to Sect. 7.

A main difference is that an extremal, braid-invariant state is not necessarily neutral, nor is the corresponding algebra necessarily a factor. It is interesting that the de Finetti theorem suggests new methods to prove whether the von-Neumann algebra $\overline{P F}_{\infty}^{\varphi}$ is a factor. Finally in Theorem 14 we characterize the extremal nature of product states (an inverse de Finetti theorem).

1.3. Organization. In Sect. 2 we define parafermion algebras and their diagrammatic representation which we call parafermion planar para algebras (PAPPA). In Sect. 3 we introduce the four-string braid group $\mathbb{B}_{\infty}$ acting on the parafermion algebra. We describe the braid using its diagrammatic representation in the PAPPA model. In Sect. 4 we introduce braid-invariant states, the action of shifts, the tail algebra, and conditional expectaions onto the tail algebra. We also introduce the neutral part of the tail algebra, which equals the center of the parafermion algebra. We derive the independence of the conditional expectation onto the tail algebra. In Sect. 6 we prove the de Finetti theorem for parafermion algebras in the case that $d$ is square free. We show that the center of parafermion algebra is equal to the tail algebra of the parafermion alegbra and that the tail algebra only consists of neutral elements. In Sect. 7 we generalize our de Finetti theorem to the case that $d$ is not square free. In this case, the tail algebra does not equal the center of the parafermion algebra, and the tail algebra contains non-neutral elements. We characterize the tail algebra for extremal, braid-invariant states.

\section{Parafermion Algebras and the PAPPA Model}

The parafermion algebra $P F_{m}$ of degree $d$ is the $\mathbb{Z}_{d}$-graded $*$-algebra generated by $\left\{c_{j}\right\}_{j=1}^{m}$, with $m$ possibly infinite. We denote the degree of a monomial $A \in P F_{m}$ by $\operatorname{deg}(A) \in \mathbb{Z}_{d}$. Since it is often useful to regard the degree as a physical "charge," we sometimes use this term interchangeably with "degree." 
The generators $c_{j}$ of the algebra are called parafermions and satisfy the canonical parafermion relations (CPRs)

$$
c_{j} c_{k}=q c_{k} c_{j}, \text { for } j<k, \quad c_{j}^{d}=I, \quad c_{j}^{*}=c_{j}^{-1},
$$

where $q=e^{\frac{2 \pi i}{d}}$, and $i=\sqrt{-1}$. Then $P F_{2 m}$ is isomorphic to the tensor product of $m$-copies of $\mathbb{M}_{d}(\mathbb{C})$ through the "Jordan-Wigner" transformation,

$$
P F_{2 m} \cong \otimes_{k=1}^{m} \mathbb{M}_{d}(\mathbb{C}) .
$$

Therefore, the parafermion algebra $P F_{\infty}:=\lim _{m \rightarrow \infty} P F_{m}$ is isomorphic to the infinite tensor product of $\mathbb{M}_{d}(\mathbb{C})$,

$$
P F_{\infty} \cong \otimes_{k=1}^{\infty} \mathbb{M}_{d}(\mathbb{C}) \text {. }
$$

According to the charge in the parafermion algebra, it can be decomposed by charge as $P F_{\infty}=\oplus_{k=0}^{d-1} P F_{\infty}^{k}$, where

$$
P F_{\infty}^{k}=\left\{x \in P F_{\infty} \mid \operatorname{deg}(x)=k\right\} .
$$

The charge-zero subalgebra $P F_{\infty}^{0}$ is called the neutral subalgebra.

We use a pictorial representation for the element $c_{j}^{m}$ introduced in [JL17], where the algebra is called PAPPA. We represent $c_{j}^{m}$ by inserting the label $m$ on the $j$ th string (numbered from left to right). We place the label of the string position above or below the string, and we omit that label in case this will cause no confusion. We interpret $m$ as a $\mathbb{Z}_{d}$-valued charge, so we also call PAPPA a "charged-string model." The correspondence between parafermion operators and pictures is:

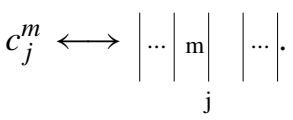

Multiplication is designated from top to bottom, corresponding to algebraic factors written from right to left. In the PAPPA model, the charged strings satisfy the following relations,

$$
\begin{array}{cc}
\text { Multiplication: } & \begin{array}{c}
m \\
n
\end{array}=m+n|, \quad d|=|=0| . \\
\text { Para isotopy: } & \left.n|| \ldots\right|^{m}\left|=q^{m n}\right||\ldots|_{m} .
\end{array}
$$

$$
\text { Twisted product: } \quad n|| \cdots|m|:=\zeta^{m n} n|| \cdots|m| .
$$

Here $\zeta$ is a chosen square root of $q$ such that $\zeta^{d^{2}}=1$ [JL17,JLW18].

\section{The Four-String Braid Group $\mathbb{B}_{\infty}$}

We consider the four-string braid group generated by exchanges of pairs of adjacent parafermions; in quantum information this corresponds to braiding adjacent qudits. 
3.1. Four string braids. The algebra generated by two consecutive generators $\left(c_{j}, c_{j+1}\right)$ is isomorphic to $\mathbb{M}_{d}(\mathbb{C})$; in other words $P F_{2} \cong \mathbb{M}_{d}(\mathbb{C})$. Motivated by this picture, we consider the action of braid group $\mathbb{B}_{\infty}$ on the pairs of the generators $\left(c_{2 j-1}, c_{2 j}\right)$. In particular, for the parafermion algebra $P F_{2 m}$, we introduce the braid group

$$
\mathbb{B}_{2 m}=\left\langle b_{1}, b_{2}, \ldots, b_{m-1}\right\rangle,
$$

that is generated by $m-1$ four-string braids. The braid $b_{j}$ intertwines the $(2 j-1)$ th and $(2 j)$ th strings with the $(2 j+1)$ th and $(2 j+2)$ th strings. The following picture represents this (negative) four-string braid action:

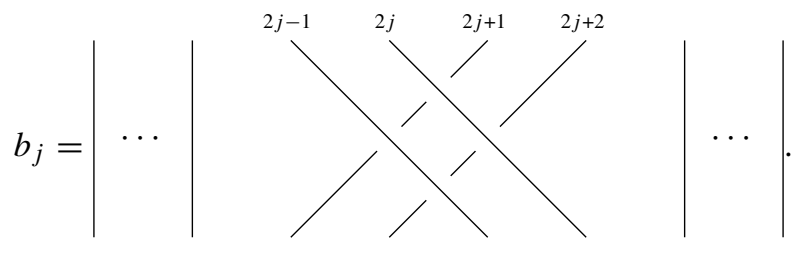

Proposition 3. Each four-string braid $b_{j}$ is an element of $P F_{\infty}$. The generators $b_{j} \in$ $\mathbb{B}_{\infty}$ satisfy the double-braid relations:

$$
\begin{aligned}
b_{j} b_{k} & =b_{k} b_{j}, \quad \text { if }|j-k|>1, \\
b_{j} b_{k} b_{j} & =b_{k} b_{j} b_{k}, \quad \text { if }|j-k|=1 .
\end{aligned}
$$

Proof. The negative four-string braid $b_{j}$ is the product of four two-string braids,

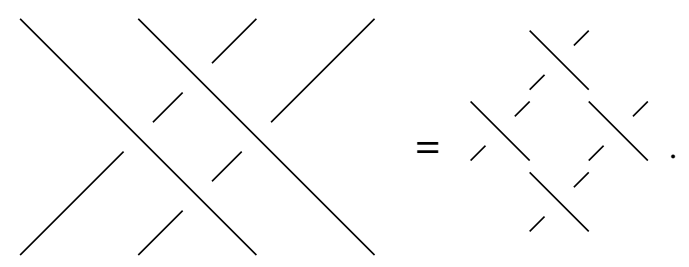

Each two-string braid can be expressed in terms of the generators of the parafermion algebra, as shown in formula (8.1) of [JL17],

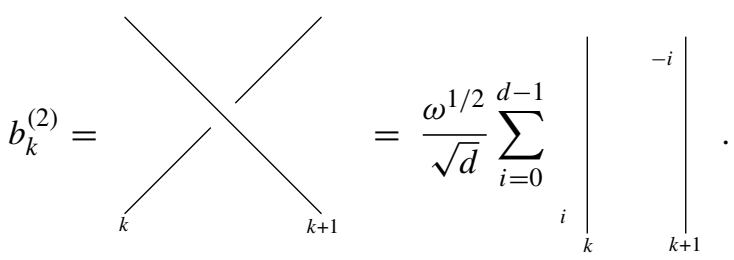

Here $\omega=\frac{1}{\sqrt{d}} \sum_{j=0}^{d-1} \zeta^{j^{2}}$ is shown to be a phase in Proposition 2.15 of [JL17]. As a consequence the two-string braid $b_{k}^{(2)}$ is a unitary, namely $b_{j}^{(2) *} b_{j}^{(2)}=I$, and hence so is the four-string braid $b_{j}$. Also the left-most single braid $b_{2 j-1}^{(2)}$ and the right-most single braid $b_{2 j+1}^{(2)}$ illustrated in (10) commute, so their relative vertical order does not matter. 
This decomposition shows that $b_{j}$ is in the algebra generated by the four parafermions $c_{2 j-1}, c_{2 j}, c_{2 j+1}, c_{2 j+2}$.

The double-braid relations (9) are evident from the picture representation of the double braid, and the fact that the single braid satisfies the three Reidermeister moves, see $\S 8$ in [JL17] and $§ 3.7$ in [JLW18].

3.2. Action of double braids on parafermions. The natural action of the double braids $\mathbb{B}_{2 m}$ on the parafermion algebra $P F_{2 m}$ is the adjoint action $\operatorname{Ad}\left(b_{j}\right) \in \operatorname{Aut}\left(P F_{2 m}\right)$. The adjoint action exchanges the pair $\left(c_{2 j-1}, c_{2 j}\right)$ with the pair $\left(c_{2 j+1}, c_{2 j+2}\right)$. Thus

$$
A d\left(b_{j}\right)\left(c_{2 j-1}^{m} c_{2 j}^{n}\right)=b_{j}\left(c_{2 j-1}^{m} c_{2 j}^{n}\right) b_{j}^{-1}=c_{2 j+1}^{m} c_{2 j+2}^{n} .
$$

The diagram that corresponds to this action is:

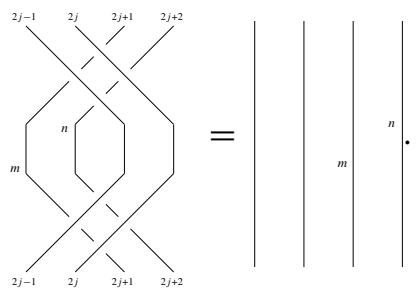

This equality combines the second Reidermeister move for the braid, as well as the fact that charges pass freely under a braid, see Theorem 8.2 in [JL17]. In a similar manner, one can analyze the case with charges on all four strings. The composition of braids translates a sequence of qudits; for example, $A d\left(b_{j-1} b_{j}\right)$ acting on the $j-1$ and $j$ qudit spaces, tensored with the identity on the qudit $j+1$, can be pictured as

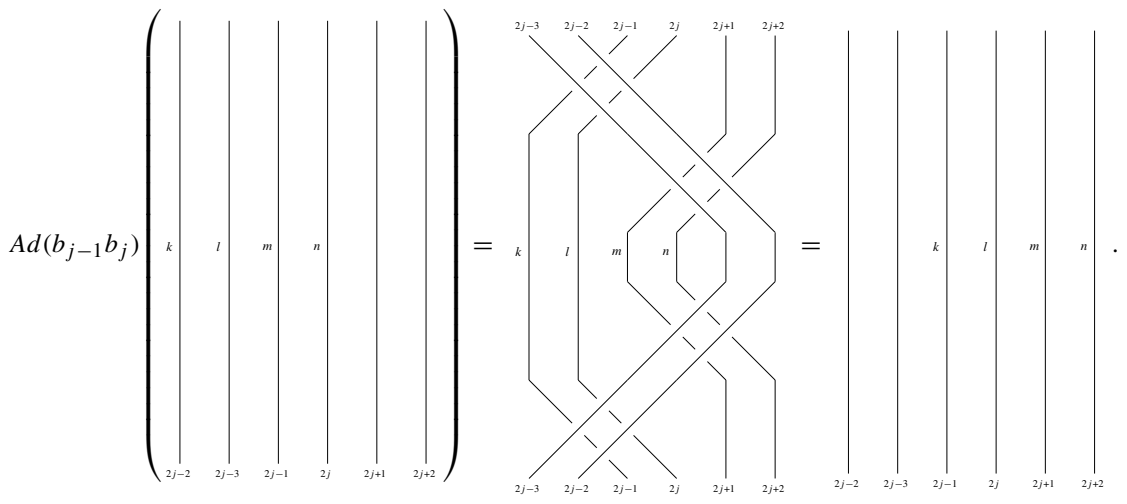

3.3. States and automorphisms. A state $\varphi$ on $P F_{\infty}$ yields by the GNS construction a Hilbert space $\mathscr{H}$, a $*$-representation $\pi$ of $P F_{\infty}$ on $\mathscr{H}$, and a cyclic vector $\Omega$ such that $\varphi(x)=\langle\Omega, \pi(x) \Omega\rangle_{\mathscr{H}}$. For simplicity, we denote $\pi(x)$ acting on $\mathscr{H}$ by $x$. We also use $x$ to denote an element of the von Neumann algebra $\overline{P F}_{\infty}^{\varphi}$ on $\mathscr{H}$ obtained by closing $P F_{\infty} / \mathscr{N}$ in the sesquilinear form $\langle x, y\rangle=\varphi\left(x^{*} y\right)$ arising from $\varphi$ with null space $\mathscr{N}$.

If the state $\varphi$ is invariant under a $*$-automorphism $\sigma$ of $P F_{\infty}$, this determines a $*$ automorphism (that we also denote as $\sigma$ ) on $\overline{P F}_{\infty}^{\varphi}$ and an isometry $U$ on $\mathscr{H}$, that leaves $\Omega$ invariant, and such that $\sigma(x) \Omega=U x \Omega$. 
3.4. Braid-invariance and shift invariance. We are especially concerned with shifts of pairs of generators of the parafermion algebra, as they correspond to the action of the four-string braids. Define the double shift $\alpha \in \operatorname{End}\left(P F_{\infty}\right)$ by

$$
\alpha\left(c_{j}\right)=c_{j+2}, \text { for all } j \in \mathbb{N} .
$$

The picture for the double shift is:

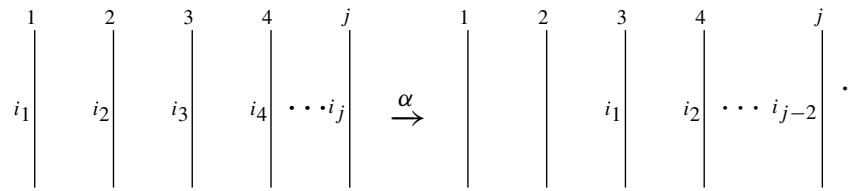

Let $\left(\overline{P F}_{\infty}^{\varphi}\right)^{\alpha}$ denote the fixed point algebra of $\overline{P F}_{\infty}^{\varphi}$ under the shift

$$
\left(\overline{P F}_{\infty}^{\varphi}\right)^{\alpha}:=\left\{x \in \overline{P F}_{\infty}^{\varphi} \mid \alpha(x)=x\right\} .
$$

A state $\varphi$ on $P F_{\infty}$ will be called $\alpha$-shift-invariant if

$$
\varphi=\varphi \circ \alpha .
$$

Let $S_{\alpha}$ denote the set of $\alpha$-shift-invaraiant states on $P F_{\infty}$.

Similarly we say that the state $\varphi$ on $P F_{\infty}$ is braid-invariant if it is invariant under the adjoint action of the braid group $\mathbb{B}_{\infty}$,

$$
\varphi=\varphi \circ \operatorname{Ad}(b),
$$

for any $b \in \mathbb{B}_{\infty}$. Let $S_{\mathbb{B}_{\infty}}$ denote the set of $\mathbb{B}_{\infty}$-invariant states on $P F_{\infty}$.

Let $\left(\overline{P F}_{\infty}^{\varphi}\right)^{\mathbb{B}_{\infty}}$ denote the fixed point algebra of $\overline{P F}_{\infty}^{\varphi}$ under the adjoint action of the braid group $\mathbb{B}_{\infty}$,

$$
\left(\overline{P F}_{\infty}^{\varphi}\right)^{\mathbb{B}_{\infty}}:=\left\{x \in \overline{P F}_{\infty}^{\varphi} \mid A d(\sigma)(x)=x, \forall \sigma \in \mathbb{B}_{\infty}\right\} .
$$

Proposition 4. For $x \in \overline{P F}_{\infty}^{\varphi}$, the strong limit of consecutive braidings exists. It equals to the shift of $x$,

$$
\alpha(x)=\text { st. }-\lim _{n \rightarrow \infty} A d\left(b_{1} b_{2} \ldots b_{n}\right)(x) \in \overline{P F}_{\infty}^{\varphi} .
$$

Any braid-invariant state is also $\alpha$-shift-invariant, $S_{\mathbb{B}_{\infty}} \subset S_{\alpha}$.

Proof. Any element $x \in P F_{2 m}$ satisfies $A d\left(b_{1} b_{2} \ldots b_{m}\right)(x)=\alpha(x)$, and furthermore $A d\left(b_{1} b_{2} \ldots b_{n}\right)(x)=A d\left(b_{1} b_{2} \ldots b_{m}\right)(x)$ for $n>m$. Thus (20) holds on this dense subalgebra.

If $x_{j} \in P F_{2 m_{j}}$ converges strongly to $x \in \overline{P F}_{\infty}^{\varphi}$, we claim that $\alpha\left(x_{j}\right)$ converges strongly to a limit that we denote $\alpha(x)$. Let $B_{\ell}=b_{1} \cdots b_{\ell}$ denote the unitary transformation implementing this element of the braid group on the GNS Hilbert space $\mathscr{H}$. Choose $y \in P F_{k}$, and without loss of generality, let $k<j<j^{\prime}$. Then

$$
\begin{aligned}
\left\|\left(\alpha\left(x_{j}\right)-\alpha\left(x_{j^{\prime}}\right)\right) y\right\|_{\mathscr{H}}^{2} & =\|\left(B_{m_{j^{\prime}}}\left(x_{j}-x_{j^{\prime}}\right) B_{m_{j^{\prime}}}^{*} y \|_{\mathscr{H}}^{2}\right. \\
& =\varphi\left(y^{*} B_{m_{j^{\prime}}}\left(x_{j}-x_{j^{\prime}}\right)^{*}\left(x_{j}-x_{j^{\prime}}\right) B_{m_{j^{\prime}}}^{*} y\right) \\
& =\varphi\left(\tilde{y}^{*}\left(x_{j}-x_{j^{\prime}}\right)^{*}\left(x_{j}-x_{j^{\prime}}\right) \tilde{y}\right) .
\end{aligned}
$$


Here we use the invariance of $\varphi$ under the braid group and the fact that $\tilde{y}=B_{m_{j^{\prime}}}^{*} y B_{m_{j^{\prime}}}$ is independent of $j^{\prime}$ for $k<j^{\prime}$. Thus as a consequence of the strong convergence of $x_{j}$, the difference (21) converges to zero as $j \rightarrow \infty$. As the $y$ range over a dense set of $\overline{P F}_{\infty}^{\varphi}$ this verifies (20). It also shows that $\varphi$ is $\alpha$-shift-invariant, for

$$
\varphi(\alpha(x))=\lim _{j} \varphi\left(\alpha\left(x_{j}\right)\right)=\lim _{j} \varphi\left(B_{m_{j}} x_{j} B_{m_{j}}^{*}\right)=\lim _{j} \varphi\left(x_{j}\right)=\varphi(x) .
$$

\section{The Tail Algebra for Parafermions}

We give the basic definitions of braid-invariance and the tail algebra for parafermions, and we derive some general properties.

4.1. Fundamental concepts. Neutral states The state $\varphi$ on $P F_{\infty}$ is called neutral, if it vanishes on elements with non-zero charge, namely $\varphi(x)=0$ for all $x$ with $\operatorname{deg}(x) \neq 0$. The tail algebra Let $\varphi$ be a state on $P F_{\infty}$, and let $H_{\varphi}$ denote the Hilbert space obtained by the GNS construction. Let $\overline{P F}_{\infty}^{\varphi}$ be the von Neumann algebra generated by the representation of $P F_{\infty}$ on $H_{\varphi}$. Define the tail algebra $P F^{T}$ of the parafermion algebra as

$$
P F^{T}=\bigcap_{n} \alpha^{n}\left(\overline{P F}_{\infty}^{\varphi}\right)
$$

Conditional expectation Given an algebra $\mathscr{A}$ and a subalgebra $\mathscr{B} \subset \mathscr{A}$, a $\mathscr{B}-\mathscr{B}$ bimodule linear map $E: \mathscr{A} \rightarrow \mathscr{B}$ is a conditional expectation if for all $a \in \mathscr{A}$ and $b, b_{1}, b_{2} \in \mathscr{B}$,

$$
E(\mathscr{A})=\mathscr{B}, \quad E(b)=b, \quad \text { and } \quad E\left(b_{1} a b_{2}\right)=b_{1} E(a) b_{2} .
$$

Charge in the tail algebra To decompose the tail algebra according to the charge, define charge of an element in the tail algebra to be compatible with the charge of elements in the parafermion algebra.

4.2. Properties of the tail algebra. The tail algebra can be characterized using the strong operator topology (SOT). For the shift $\alpha$ defined by (14) on $P F_{\infty}$, define the shiftaveraging transformation

$$
\mathfrak{S}_{k}=\frac{\alpha+\alpha^{2}+\cdots+\alpha^{k}}{k} .
$$

Given a state $\varphi$, we also have the corresponding $\alpha$ and $\mathfrak{S}_{k}$ on $\overline{P F}_{\infty}^{\varphi}$.

Proposition 5. Let $\varphi$ be an $\alpha$-shift-invariant state on $P F_{\infty}$, and let $x \in \overline{P F}_{\infty}^{\varphi}$. Then $\mathfrak{S}_{k}(x)$ converges strongly to an element in the tail,

$$
E_{T}(x)=\text { st. }-\lim _{k \rightarrow \infty} \mathfrak{S}_{k}(x) .
$$

The map $E_{T}$ defines a normal, unital conditional expectation from $\overline{P F}_{\infty}^{\varphi}$ to $P F^{T}$. Also

$$
E_{T}(x)=E_{T}(\alpha(x)) \text {, and }\left(E_{T}(x)\right)^{*}=E_{T}\left(x^{*}\right) .
$$


Proof. Clearly $E_{T}(I)=I$, and

$$
\text { st.- } \lim _{k} \frac{\alpha(x)-\alpha^{(k+1)}(x)}{k}=0 .
$$

So if $E_{T}(x)=$ st.- $\lim _{k \rightarrow \infty} \mathfrak{S}_{k}(x)$ exists, then $E_{T}(\alpha(x))=E_{T}(x)$, and the limit is in the tail algebra $P F^{T}$. As $\left\|\mathfrak{S}_{k}(x)\right\| \leqslant\|x\|$, it is sufficient to show that $\lim _{k} \mathfrak{S}_{k}(x) A \Omega$ exists for all monomials $A \in P F_{2 m}$, with $m \geqslant 1$.

We first establish the limit when $x$ is a monomial in $P F_{2 m}$, so that for any $k>m$, and for $r=-\operatorname{deg}(x) \operatorname{deg}(A)$,

$$
\alpha^{k}(x) A=q^{r} A \alpha^{k}(x) \text {. }
$$

At most $2 m$ terms differ in the sums defining $\mathfrak{S}_{k}(x) A$ and $q^{r} A \mathfrak{S}_{k}(x)$, so ${ }^{1}$

$$
\left|\mathfrak{S}_{k}(x) A \Omega-q^{r} A \mathfrak{S}_{k}(x) \Omega\right| \leqslant \frac{2 m}{k}\|A\|\|x\||\Omega| \rightarrow_{k \rightarrow \infty} 0 .
$$

Since $\varphi$ is $\alpha$-shift-invariant, there is a unitary $U_{\alpha}$ on $H_{\varphi}$ that implements $\alpha$ and leaves $\Omega$ invariant, namely

$$
U_{\alpha} A \Omega=\alpha(A) \Omega
$$

Then

$$
\mathfrak{S}_{k}(x) \Omega=\left(\frac{U_{\alpha}+U_{\alpha}^{2}+\cdots+U_{\alpha}^{k}}{k}\right) x \Omega .
$$

Here the unitary $U_{\alpha}$ implements $\alpha$ and leaves $\Omega$ invariant. Now we use the von Neumann mean ergodic theorem, see page 407 of [RS55], to conclude that the vectors (30) converge strongly as $k \rightarrow \infty$. Hence any $x \in P F_{2 m}$ satisfies

$$
E_{T}(x) \Omega=\lim _{k} \mathfrak{S}_{k}(x) \Omega, \text { and } E_{T}(x) A \Omega=q^{r} A E_{T}(x) \Omega .
$$

Any operator in $P F_{2 m}$ is a finite sum of monomials, so the limit (25) exists for operators in $P F_{\infty}$.

Now we show that the limit (25) extends to all $x_{0} \in \overline{P F}_{\infty}^{\varphi}$. By Kaplansky density theorem, there are $x_{j} \in P F_{2 j}, j=1,2, \ldots$, such that $\left\|x_{j}\right\| \leqslant\left\|x_{0}\right\|$ and st.- $\lim _{j} x_{j}=$ $x_{0}$. Let $\ell \in \mathbb{Z}_{d}$ and $y_{j}=\alpha\left(x_{j}\right)$. Define

$$
y_{j, \ell}=\frac{1}{d} \sum_{k \in \mathbb{Z}_{d}} q^{k \ell} c_{1}^{-k} y_{j} c_{1}^{k}
$$

Then $y_{j}=\sum_{\ell \in \mathbb{Z}_{d}} y_{j, \ell}$, with $\operatorname{deg}\left(y_{j, \ell}\right)=\ell$, and they satisfy

$$
\left\|y_{j, \ell}\right\| \leqslant\left\|y_{j}\right\| \leqslant\left\|x_{0}\right\|, \quad \text { and } \quad \text { st. } \lim _{j} y_{j, \ell}=y_{0, \ell}
$$

Whenever $k>m$,

$$
\alpha^{k}\left(y_{j, \ell}\right) A=q^{-\operatorname{deg}(A) \ell} A \alpha^{k}\left(y_{j, \ell}\right) .
$$

Arguing as above, we infer that

$$
\left|\mathfrak{S}_{k}\left(y_{j, \ell}\right) A \Omega-q^{-\operatorname{deg}(A) \ell} A \mathfrak{S}_{k}\left(y_{j, \ell}\right) \Omega\right| \leqslant \frac{2 m}{k}\|A\|\left\|x_{0}\right\||\Omega| .
$$

\footnotetext{
1 We use $\|\cdot\|$ to denote the norm of an operator and $|\cdot|$ to denote the norm of a vector.
} 
Furthermore $\mathfrak{S}_{k}\left(y_{0, \ell}\right)$ has a strong limit $E_{T}\left(y_{0, \ell}\right)$, such that

$$
\begin{aligned}
E_{T}\left(y_{j, \ell}\right) \Omega & =\lim _{k} \mathfrak{S}_{k}\left(y_{j, \ell}\right) \Omega, \\
E_{T}\left(y_{j, \ell}\right) A \Omega & =q^{-\operatorname{deg}(A) \ell} A E_{T}\left(y_{j, \ell}\right) \Omega .
\end{aligned}
$$

Therefore $E_{T}\left(y_{0}\right)$ exists. By (27), $E_{T}\left(x_{0}\right)$ exists and

$$
E_{T}\left(x_{0}\right)=E_{T}\left(y_{0}\right)=\sum_{\ell \in \mathbb{Z}_{d}} E_{T}\left(y_{0, \ell}\right) .
$$

Suppose $\left\{x_{m}\right\}$ is a sequence in the unit ball of $\overline{P F}_{\infty}^{\varphi}$, and st.- $\lim _{j} x_{j}=x_{0}$. To show that $E_{T}$ is normal, it is sufficient to show that $\lim _{j} E_{T}\left(x_{j}\right)=E_{T}\left(x_{0}\right)$. Define $y_{j, \ell}$ as above, so the properties above remain true. Moreover,

$$
\begin{aligned}
\left|\mathfrak{S}_{k}\left(y_{0, \ell}\right) \Omega-\mathfrak{S}_{k}\left(y_{j, \ell}\right) \Omega\right| & =\left|\left(\frac{U_{\alpha}+U_{\alpha}^{2}+\cdots+U_{\alpha}^{k}}{k}\right)\left(y_{0, \ell}-y_{j, \ell}\right) \Omega\right| \\
& \leqslant\left|\left(y_{0, \ell}-y_{j, \ell}\right) \Omega\right| .
\end{aligned}
$$

So

$$
\begin{aligned}
\left|\left(E_{T}\left(y_{0, \ell}\right)-E_{T}\left(y_{j, \ell}\right)\right) \Omega\right| & \leqslant\left|\left(y_{0, \ell}-y_{j, \ell}\right) \Omega\right|, \\
\left|\left(E_{T}\left(y_{0, \ell}\right)-E_{T}\left(y_{j, \ell}\right)\right) A \Omega\right| & =\left|A\left(E_{T}\left(y_{0, \ell}\right)-E_{T}\left(y_{j, \ell}\right)\right) \Omega\right| \\
& \leqslant\|A\|\left|\left(y_{0, \ell}-y_{j, \ell}\right) \Omega\right| .
\end{aligned}
$$

Therefore,

$$
\begin{aligned}
\text { st.- } \lim _{j} E_{T}\left(y_{j, \ell}\right) & =E_{T}\left(y_{0, \ell}\right), \quad \text { st.- } \lim _{j} E_{T}\left(y_{j}\right)=E_{T}\left(y_{0}\right), \quad \text { and } \\
\text { st.- } \lim _{j} E_{T}\left(x_{j}\right) & =E_{T}\left(x_{0}\right) .
\end{aligned}
$$

Finally we verify that the map $x \mapsto E_{T}(x)$ defines a conditional expectation, by checking the three defining relations in (23). We have shown the first identity in (26). Note that $\mathfrak{S}_{k}\left(x^{*}\right)=\mathfrak{S}_{k}(x)^{*}$, so taking the limit in $k$ we obtain the second identity in (26). For the third identity, consider $y_{1}, y_{2} \in P F^{T}$. Then

$$
y_{1} \mathfrak{S}_{k}(x) y_{2}=\mathfrak{S}_{k}\left(y_{1} x y_{2}\right)
$$

using the invariance of $P F^{T}$ under the shift $\alpha$. As a consequence the $k \rightarrow \infty$ limits agree, so $x \mapsto E_{T}(x)$ does define a conditional expectation.

Remark 6. A combination of Theorem 2.2 in [GK09] and Proposition 7.3 in [K10] also shows that $E_{T}$ is a conditional expectation onto the tail algebra.

Corollary 7. Let $\varphi \in S_{\mathbb{B}_{\infty}}$, then $\varphi=\varphi \circ E_{T}$.

Proof. From Proposition 4 we infer that the state $\varphi$ is invariant under the action of $\alpha$, and from Proposition 5 we infer that $\varphi \circ E_{T}=\varphi$. 
Proposition 8. The tail algebra $P F^{T}$ is a commutative $\mathbb{Z}_{d}$-graded von Neumann algebra, with the charge- $\ell$ part denoted $P F^{T, \ell}$,

$$
P F^{T}=\bigoplus_{\ell \in \mathbb{Z}_{d}} P T^{T, \ell} .
$$

Moreover, $P T^{T, \ell}=0$ when $d \nmid \ell^{2}$.

Proof. As in the proof of Proposition 5, for any $x \in P F^{T}$, there are $y_{\ell} \in \overline{P F}_{\infty}^{\varphi}, \ell \in \mathbb{Z}_{d}$, such that

$$
\begin{gathered}
x=\alpha(x)=\sum_{\ell \in \mathbb{Z}_{d}} y_{\ell}, \\
c_{1} y_{\ell} c_{1}^{-1}=q^{\ell} y_{\ell} .
\end{gathered}
$$

Define $y_{\ell}$ to have charge $\ell$. By Proposition 5,

$$
\begin{aligned}
x & =\sum_{\ell \in \mathbb{Z}_{d}} E_{T}\left(y_{\ell}\right), \\
\text { and } \quad c_{1} z \ell c_{1}^{-1} & =q^{\ell} E_{T}\left(y_{\ell}\right) . \\
c_{1} x c_{1}^{-1} & =\sum_{\ell \in \mathbb{Z}_{d}} q^{\ell} E_{T}\left(y_{\ell}\right) .
\end{aligned}
$$

Therefore, the conjugation by the first parafermion generator $c_{1}$ defines an automorphism on the tail algebra. So if $x$ has charge $\ell$, there are operators $y_{j} \in P F_{2(j+1)}$ with charge $\ell$, for $j \geqslant 1$, such that

$$
\text { st.- } \lim _{j \rightarrow \infty} y_{j}=x
$$

Then

$$
\begin{array}{r}
\text { st.- } \lim _{j \rightarrow \infty} \alpha^{k}\left(y_{j}\right)=x, \\
\text { st.- } \lim _{j \rightarrow \infty} \alpha^{k}\left(y_{j}^{*}\right)=x^{*} .
\end{array}
$$

Note that

$$
\alpha^{k}\left(y_{j}\right) y_{j}^{*}=q^{\ell^{2}} y_{j}^{*} \alpha^{k}\left(y_{j}\right), \forall k>j+1 .
$$

So $x y_{j}^{*}=q^{\ell^{2}} y_{j}^{*} x$. Then $x \alpha^{k}\left(y_{j}^{*}\right)=q^{\ell^{2}} \alpha^{k}\left(y_{j}^{*}\right) x$, and

$$
x x^{*}=q^{\ell^{2}} x^{*} x
$$

Both $x x^{*}$ and $x^{*} x$ are positive operators, so $q^{\ell^{2}}=1$, namely $d \mid \ell^{2}$.

If $z \in P F_{T}$ has charge $\ell^{\prime}$, then similarly we have that $d \mid\left(\ell^{\prime}\right)^{2}$ and

$$
x z=q^{-\ell \ell^{\prime}} z x .
$$

Then $d^{2} \mid\left(\ell \ell^{\prime}\right)^{2}$. So $d \mid \ell \ell^{\prime}$, and $x z=z x$. Therefore $P F^{T}$ is commutative. 
Proposition 9. Given a braid-invariant state $\varphi \in S_{\mathbb{B}_{\infty}}$ and the corresponding tail algebra $P F^{T}$,

$$
P F^{T}=\left(\overline{P F}_{\infty}^{\varphi}\right)^{\alpha}=\left(\overline{P F}_{\infty}^{\varphi}\right)^{\mathbb{B}_{\infty}} .
$$

This result works in a general situation, see Theorem 0.3 in [GK09]. We give a quick proof for braided parafermions here.

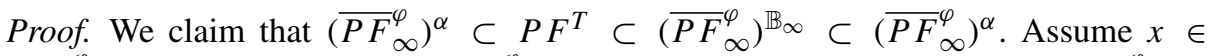
$\left(\overline{P F}_{\infty}^{\varphi}\right)^{\alpha}$, then $x=\alpha^{n}(x) \in \alpha^{n}\left(\overline{P F}_{\infty}^{\varphi}\right)$ for any $n$, and in particular $x \in \bigcap_{n} \alpha^{n}\left(\overline{P F}_{\infty}^{\varphi}\right)=$ $P F^{T}$. For any $x \in P F^{T}$, one has $A d\left(b_{n}\right)(x)=x$ for any $n$ as $x \in \alpha\left(\overline{P F}_{\infty}^{\varphi}\right)$. Thus, $x \in\left(\overline{P F}_{\infty}^{\varphi}\right)^{\mathbb{B}_{\infty}}$, i.e., $P F^{T} \subset\left(\overline{P F}_{\infty}^{\varphi}\right)^{\mathbb{B}_{\infty}}$. Moreover, $\left(\overline{P F}_{\infty}^{\varphi}\right)^{\mathbb{B}_{\infty}} \subset\left(\overline{P F}_{\infty}^{\varphi}\right)^{\alpha}$ follows from Proposition 4.

Given a state $\varphi \in S_{\mathbb{B}_{\infty}}$, let $P F^{T, 0}$ denote the neutral subalgebra of $P F^{T}$, and let $Z\left(\overline{P F}_{\infty}^{\varphi}\right)$ denote the center of the von Neumann algebra $\overline{P F}_{\infty}^{\varphi}$.

Proposition 10. Let $\varphi \in S_{\mathbb{B}_{\infty}}$. Then

$$
P F^{T, 0}=Z\left(\overline{P F}_{\infty}^{\varphi}\right) \subset P F^{T} .
$$

Proof. Any neutral $x \in P F^{T}$ commutes with all elements in $P F_{\infty}$, and therefore commutes with all elements in $\overline{P F}_{\infty}^{\varphi}$. Hence $P F^{T, 0} \subset Z\left(\overline{P F}_{\infty}^{\varphi}\right)$. Furthermore, we infer from Proposition 3 that any braid $b_{j} \in P F_{\infty}$, and also $b_{j} \in \overline{P F}_{\infty}^{\varphi}$. So if $x \in$ $Z\left(\overline{P F}_{\infty}^{\varphi}\right)$, one has $b_{j} x=x b_{j}$, and $x$ is invariant under the adjoint action of every $b_{j}$, and $x \in\left(\overline{P F}_{\infty}^{\varphi}\right)^{\mathbb{B}} \infty$. Thus we infer from Proposition 9 that $x \in\left(\overline{P F}_{\infty}^{\varphi}\right)^{\alpha}=P F^{T}$, so $P F^{T, 0} \subset Z\left(\overline{P F}_{\infty}^{\varphi}\right) \subset P F^{T}$.

If $x \in Z\left(\overline{P F}_{\infty}^{\varphi}\right)$, then $c_{1} E_{T}(x)=E_{T}(x) c_{1}$. Since $x \in Z\left(\overline{P F}_{\infty}^{\varphi}\right) \subset P F^{T}$, $c_{1} E_{T}(x) c_{1}^{-1}=q^{\operatorname{deg}\left(E_{T}(x)\right)} E_{T}(x)$. So $\operatorname{deg}\left(E_{T}(x)\right)=0$. Hence $Z\left(\overline{P F}_{\infty}^{\varphi}\right) \subset P F^{T, 0}$, and $P F^{T, 0}=Z\left(\overline{P F}_{\infty}^{\varphi}\right)$.

Let $I$ be a subset $I \subset \mathbb{N}$, and let $P F_{I}$ denote the parafermion algebra generated by the $c_{i}$ with $i \in I$. For two subsets $I, J \subset \mathbb{N}$, let $I<J$ means that for $i<j$ for all $i \in I, j \in J$. Clearly if $x \in P F_{I}$ and $y \in P F_{J}$ with $I<J$, then

$$
x y=q^{\operatorname{deg}(x) \operatorname{deg}(y)} y x .
$$

Proposition 11. Let $\varphi \in S_{\mathbb{B}_{\infty}}$, and let $x \in P F_{I}, y \in P F_{J}$, where $I<J$ or $J<I$ are finite subsets of $\mathbb{N}$. Then $E_{T}(x y)=E_{T}(x) E_{T}(y)$. Likewise, if $x_{i} \in P F_{I_{i}}$ for increasing intervals $I_{i}<I_{j}$ for $i<j$, then

$$
E_{T}\left(x_{1} \cdots x_{k}\right)=\prod_{i=1}^{k} E_{T}\left(x_{i}\right) .
$$

Such independence is called order tail-independence in Ref. [K10], where a stronger notion of independence, called full tail-independence, has also been proposed and investigated. It was shown in Theorem 8.1 in [K10] that spreadability implies tail-independence. Here we give a quick proof for braided parafermions. 
Proof. If $I<J$, there exists $\sigma_{n} \in \mathbb{B}_{\infty}$ such that $\sigma_{n}(x y)=x \alpha^{n}(y)$. Thus using from Proposition 9 and the invariance of $P F^{T}$ under braids,

$$
E_{T}(x y)=E_{T}\left(\sigma_{n}(x y)\right)=E_{T}\left(x \alpha^{n}(y)\right)=E_{T}\left(x \mathfrak{S}_{k}(y)\right)=E_{T}\left(x E_{T}(y)\right) .
$$

Then using (26), the $\alpha$-shift-invariance of $P F^{T}$ given in Proposition 9, and the fact that $P F^{T}$ is an algebra, we infer for $n, k \in \mathbb{N}$ that

$$
\begin{aligned}
E_{T}(x y) & =E_{T}\left(\alpha^{n}\left(x E_{T}(y)\right)\right)=E_{T}\left(\alpha^{n}(x) E_{T}(y)\right)=E_{T}\left(\mathfrak{S}_{k}(x) E_{T}(y)\right) \\
& =E_{T}\left(E_{T}(x) E_{T}(y)\right)=E_{T}(x) E_{T}(y) .
\end{aligned}
$$

If $x$ and $y$ have a given degree, then $\operatorname{deg}\left(E_{T}(x)\right)=\operatorname{deg}(x)$. So if $J<I$ and $x y=\left(y^{*} x^{*}\right)^{*}$, we obtain from the previous case,

$$
E_{T}(x y)=E_{T}\left(\left(y^{*} x^{*}\right)^{*}\right)=E_{T}\left(y^{*} x^{*}\right)^{*}=\left(E_{T}(y)^{*} E_{T}(x)^{*}\right)^{*}=E_{T}(x) E_{T}(y) .
$$

The general case for two elements follows by linearity for $x$ and $y$ a sum of components with definite degree. The case for $k$ ordered elements follows by iteration of the twoelement case.

Proposition 12. Let $\varphi$ be an extremal state in $S_{\mathbb{B}_{\infty}}$, then $P F^{T, 0}=\mathbb{C}$.

Proof. We show that if $P F^{T, 0}$ is not trivial, then $\varphi$ is not extremal. If $\operatorname{dim}\left(P F^{T, 0}\right) \neq 1$, there exists a non-trivial projection $P \in P F^{T, 0}$. By Proposition $10, P F^{T, 0}=Z\left(\overline{P F}_{\infty}^{\varphi}\right)$. If $\varphi(P)=0$, then $0 \leqslant \varphi\left(x^{*} P x\right)=\varphi\left(P x^{*} x\right) \leqslant \sqrt{\varphi(P) \varphi\left(\left(x^{*} x\right)^{2}\right)}=0$. So $\varphi\left(x^{*} P x\right)=$ 0 , for any $x \in \overline{P F}_{\infty}^{\varphi}$. Therefore $P=0$ in $\overline{P F}_{\infty}^{\varphi}$, a contradiction. Similarly, if $\varphi(P)=1$, then $P=I$, a contradiction. Therefore $\beta=\varphi(P) \in(0,1)$.

Proposition 9 shows $P$ is invariant under action of $\mathbb{B}_{\infty}$. Let $\varphi_{1}(\cdot)=\frac{1}{\beta} \varphi(P(\cdot))$, and let $\varphi_{2}(\cdot)=\frac{1}{1-\beta} \varphi((1-P)(\cdot))$. Then $\varphi_{1}, \varphi_{2} \in S_{\mathbb{B}_{\infty}}$ and $\varphi=\beta \varphi_{1}+(1-\beta) \varphi_{2}$, which contracts with the fact that $\varphi$ is extremal.

\section{The Inverse de Finetti Theorem}

Suppose $\mathscr{A}$ is a finite dimensional matrix algebra and $\rho$ is a state on $\mathscr{A}$. Let $\mathscr{A}^{\otimes m}=$ $\otimes_{k=1}^{m} \mathscr{A}$ be the $m$ th tensor power of $\mathscr{A}$ and $\mathscr{A}_{\infty}=\otimes_{k=1}^{\infty} \mathscr{A}$ be the infinite tensor power of $\mathscr{A}$. Let $S_{P, \mathscr{A}}$ be the states on the infinite tensor product of $\mathscr{A}$ which are invariant under the permutation group. The de Finetti theorem said that if $\varphi$ is an extremal point in $S_{P, \mathscr{A}_{\infty}}$, then $\varphi$ is the infinite product state $\prod \rho$, for some state $\rho$ on $\mathscr{A}$. It was shown by Størmer, that any symmetric product state is extremal in $S_{P, \mathscr{A}_{\infty}}$, see Theorem 2.7 in [Sto69] for a general result on $C^{*}$-algebras.

The tensor product of states on $\mathscr{A}$ is called a product state. The symmetric product state is not an extremal point for the symmetric states on finite tensor products. We have the following extremal condition for finite symmetric product states, which can be considered as an inverse de Finetti theorem on finite tensors:

Theorem 13. Suppose $\mathscr{A}$ is a finite dimensional matrix algebra. Let $\mathscr{S}$ be the space of states on $\mathscr{A}$. For $m \geqslant 2$, take $\mathscr{A}^{\otimes m}=\otimes_{k=1}^{m} \mathscr{A}$. For a state $\phi \in \mathscr{S}$, if $\mu$ is a probability measure of $\mathscr{S}$, such that,

$$
\phi^{m}=\int_{\rho \in \mathscr{S}} \rho^{m} d \mu(\rho)
$$

on $\mathscr{A}^{\otimes m}$, then $\mu$ is the Dirac measure at $\phi$. 
Proof. Take the restriction on $\mathscr{A}^{\otimes 2}$, we have that

$$
\phi^{2}=\int_{\rho \in \mathscr{S}} \rho^{2} d \mu(\rho) .
$$

Let $D_{\rho}$ be the density matrix of $\rho$. Then

$$
D_{\phi} \otimes D_{\phi}=\int_{\rho \in \mathscr{S}} D_{\rho} \otimes D_{\rho} d \mu(\rho) .
$$

Let $P$ be the range projection of $D_{\phi}$, and $Q=I-P$, then

$$
\int_{\rho \in \mathscr{S}} Q D_{\rho} Q \otimes Q D_{\rho} Q d \mu(\rho)=0 .
$$

Therefore, $Q D_{\rho} Q=0$ for almost all $D$. So $Q D=D Q=0$, and $D=P D P$. Without loss of generality, we assume that $P=I$, then $D_{\phi}$ is invertible. Take $C_{\rho}=$ $D_{\phi}^{-1 / 2} D_{\rho} D_{\phi}^{-1 / 2}$. Then

$$
I \otimes I=\int_{\rho \in \mathscr{S}} C_{\rho} \otimes C_{\rho} d \mu(\rho) .
$$

Let $t r$ be the tracial state on $\mathscr{A}$. Then taking the trace of the tensor/product in the above formula,

$$
\begin{gathered}
\int_{\rho \in \mathscr{S}} \operatorname{tr}\left(C_{\rho}\right)^{2} d \mu(\rho)=\operatorname{tr}(I)^{2}=1, \\
\int_{\rho \in \mathscr{S}} \operatorname{tr}\left(C_{\rho}^{2}\right) d \mu(\rho)=\operatorname{tr}\left(I^{2}\right)=1 .
\end{gathered}
$$

So

$$
\int_{\rho \in \mathscr{S}}\left(\operatorname{tr}\left(C_{\rho}^{2}\right)-\operatorname{tr}\left(C_{\rho}\right)^{2}\right) d \mu(\rho)=0 .
$$

Note that the covariance of $C_{\rho}$ is

$$
\operatorname{Cov}\left(C_{\rho}\right)=\operatorname{tr}\left(C_{\rho}^{2}\right)-\operatorname{tr}\left(C_{\rho}\right)^{2}=\operatorname{tr}\left(\left(C_{\rho}-\operatorname{tr}\left(C_{\rho}\right)\right)^{2}\right) \geqslant 0 .
$$

So $\operatorname{Cov}\left(C_{\rho}\right)=0$, and $C_{\rho}=\operatorname{tr}\left(C_{\rho}\right)$ for almost all $\rho$. Then $D_{\rho}=\operatorname{tr}\left(C_{\rho}\right) D_{\phi}$. Both $D_{\rho}$ and $D_{\phi}$ have trace one, so $D_{\rho}=D_{\phi}$. Therefore $\mu$ is a Dirac measure at $\rho$.

The above proof also applies to infinite tensors and we recover the result of Størmer for matrix algebras:

Theorem 14. Suppose $\rho$ is a state on a finite dimensional matrix algebra $\mathscr{A}$. Then the product state $\prod \rho$ is extremal in $S_{P, \mathscr{A}_{\infty}}$.

Proof. By the de Finetti theorem, any extremal point in $S_{P, \mathscr{A}_{\infty}}$ is a product state $\rho^{\infty}$, for some $\rho \in \mathscr{S}$. Note that $\rho_{j} \rightarrow \rho$ weakly on $\mathscr{A}$ iff $\rho_{j}^{\infty} \rightarrow \rho^{\infty}$ weakly on $\mathscr{A}_{\infty}$. Thus 
the space of infinite symmetric product states has the same weak topology as $\mathscr{S}$. By the Choquet-Bishop-de Leeuw theorem, any state in $S_{P, \mathscr{A}_{\infty}}$ is

$$
\int_{\rho \in \mathscr{S}} \rho^{\infty} d \mu(\rho),
$$

for some probability measure $\mu$ on $\mathscr{S}$. Therefore, any convex combination is also of the above form. For a state $\phi \in \mathscr{S}$, if

$$
\phi^{\infty}=\int_{\rho \in \mathscr{S}} \rho^{\infty} d \mu(\rho),
$$

then

$$
\phi^{2}=\int_{\rho \in \mathscr{S}} \rho^{2} d \mu(\rho) .
$$

By Theorem 13, $\int_{\rho=\phi} d \mu(\rho)=1$. Therefore, $\phi^{\infty}$ is extremal in $S_{P, A_{\infty}}$.

\section{The de Finetti Theorem: Square-Free $d$, the Neutral Case}

As explained in the introduction, there are two possible outcomes according to whether the degree $d$ of the parafermion algebra is square free. In this section we investigate the square-free case. We first prove that the center of the representation of the parafermion algebra equals the tail algebra, and that the tail algebra is neutral.

Several equivalent characterization of the extremal state in $S_{\mathbb{B}_{\infty}}$ follow from these results, and we precisely characterize the corresponding braid-invariant states. We call Theorem 17 the de Finetti theorem for the parafermion algebra with square-free degree $d$.

Theorem 15. Let $d=\prod p_{i}$ be square free, and let $\varphi \in S_{\mathbb{B}_{\infty}}$ be braid invariant. Then $\varphi$ is neutral: $\varphi(x)=0$, whenever deg $(x) \neq 0$.

Proof. Denote $C_{i}^{m n}=c_{2 i-1}^{m} c_{2 i}^{n}$. We first prove that for $j<k$,

$$
\varphi\left(E_{T}\left(C_{j}^{m n}\right) E_{T}\left(C_{k}^{m n}\right)^{*}\right)=0, \quad \text { unless } m+n=0(\bmod d)
$$

Using Corollary 7, with Proposition 11, and the second identity in (26), we have

$$
\begin{aligned}
\varphi\left(C_{j}^{m n}\left(C_{k}^{m n}\right)^{*}\right) & =\varphi\left(E_{T}\left(C_{j}^{m n}\left(C_{k}^{m n}\right)^{*}\right)\right)=\varphi\left(E_{T}\left(C_{j}^{m n}\right) E_{T}\left(C_{k}^{m n}\right)^{*}\right) \\
& =\varphi\left(E_{T}\left(C_{k}^{m n}\right) E_{T}\left(C_{k}^{m n}\right)^{*}\right) \geqslant 0 .
\end{aligned}
$$

In the last line we use $\varphi \geqslant 0$, as well as $E_{T}\left(C_{j}^{m n}\right)=E_{T}\left(C_{k}^{m n}\right)$. This property follows from the first identity in (26), for

$$
E_{T}\left(C_{j}^{m n}\right)=\alpha^{k-j}\left(E_{T}\left(C_{j}^{m n}\right)\right)=E_{T}\left(\alpha^{k-j}\left(C_{j}^{m n}\right)\right)=E_{T}\left(C_{k}^{m n}\right) .
$$


On the other hand, the parafermion relations (1), and reasoning similar to the proof of (58), show that

$$
\begin{aligned}
\varphi\left(C_{j}^{m n}\left(C_{k}^{m n}\right)^{*}\right) & =q^{-(m+n)^{2}} \varphi\left(\left(C_{k}^{m n}\right)^{*} C_{j}^{m n}\right) \\
& =q^{-(m+n)^{2}} \varphi\left(E_{T}\left(\left(C_{k}^{m n}\right)^{*} C_{j}^{m n}\right)\right) \\
& =q^{-(m+n)^{2}} \varphi\left(E_{T}\left(\left(C_{k}^{m n}\right)^{*} C_{j}^{m n}\right)\right) \\
& =q^{-(m+n)^{2}} \varphi\left(E_{T}\left(C_{k}^{m n}\right)^{*} E_{T}\left(C_{j}^{m n}\right)\right) \\
& =q^{-(m+n)^{2}} \varphi\left(E_{T}\left(C_{k}^{m n}\right)^{*} E_{T}\left(C_{k}^{m n}\right)\right),
\end{aligned}
$$

where $\varphi\left(E_{T}\left(C_{k}^{m n}\right)^{*} E_{T}\left(C_{k}^{m n}\right)\right) \geqslant 0$. Comparing (58) with (59), we infer that either $q^{(m+n)^{2}}>0$, or else $\varphi\left(C_{j}^{m n}\left(C_{k}^{m n}\right)^{*}\right)=0$.

In our case $0 \leqslant m, n \leqslant d-1$. As we assume that $d$ is square free, $(m+n)^{2}=$ $0(\bmod d)$ is equivalent to $(m+n)=0(\bmod d)$. Thus $(57)$ holds.

If $m+n \neq 0 \bmod d$, we have shown in addition that

$$
\varphi\left(E_{T}\left(C_{k}^{m n}\right) E_{T}\left(C_{k}^{m n}\right)^{*}\right)=0 .
$$

So $E_{T}\left(C_{k}^{m n}\right)=0$, and

$$
\varphi\left(C_{j}^{m n}\right)=\varphi\left(C_{k}^{m n}\right)=\varphi\left(E_{T}\left(C_{k}^{m n}\right)\right)=0 .
$$

Any element $x \in P F_{\infty}$ can be expressed as the linear combination of the products $\prod_{k} C_{k}^{m_{k} n_{k}}$, where we take the product in the order of increasing $k$ from left to right. By (56) we have

$$
\varphi\left(\prod_{k} C_{k}^{m_{k} n_{k}}\right)=E_{T}\left(\prod_{k} C_{k}^{m_{k} n_{k}}\right)=\prod_{k} E_{T}\left(C_{k}^{m_{k} n_{k}}\right)=0
$$

unless each $\left(m_{k}+n_{k}\right)=0(\bmod d)$, for all $k$.

Corollary 16. Let $d=\prod p_{i}$ be square free, and let $\varphi \in S_{\mathbb{B}_{\infty}}$. Then

$$
P F^{T, 0}=Z\left(\overline{P F}_{\infty}^{\varphi}\right)=P F^{T}=\left(\overline{P F}_{\infty}^{\varphi}\right)^{\alpha}=\left(\overline{P F}_{\infty}^{\varphi}\right)^{\mathbb{B}_{\infty}}
$$

Proof. With the results of Propositions 9 and 10, we only need to show that $P F^{T, 0}=$ $P F^{T}$. For this use the fact that we have shown in Theorem 15 that the generators of the tail algebra are all neutral.

Theorem 17. (Neutral Case) Let $\varphi \in S_{\mathbb{B}_{\infty}}$ be a braid-invariant state on the parafermion algebra $P F_{\infty}$ of square-free order $d$. Then (1)-(5) are equivalent conditions:

(1) The state $\varphi$ is extremal in $S_{\mathbb{B}_{\infty}}$.

(2) The state $\varphi=\prod_{j} \rho$ is a product, where $\rho$ is a neutral state on $P F_{2}$.

(3) The neutral part of the tail algebra $P F^{T, 0}=\mathbb{C}$.

(4) The tail algebra $P F^{T}=\mathbb{C}$.

(5) The von Neumann algebra $\overline{P F}_{\infty}^{\varphi}$ is a factor. 
Proof. (1) $\Rightarrow$ (3): This is established in Proposition 12.

(4) $\Rightarrow$ (2): Since any element $x \in P F_{\infty}$ can be expressed as the linear combination of the form $\prod_{k} C_{k}^{m_{k} n_{k}}$, we only need to prove that

$$
\varphi\left(\prod_{k} C_{k}^{m_{k} n_{k}}\right)=\prod_{k} \varphi\left(C_{k}^{m_{k} n_{k}}\right)
$$

But this is a consequence of Corollary 7, which ensures that $\varphi=\varphi \circ E_{T}$, and Proposition 11, which shows that $\varphi$ factors on non-overlapping elements of the tail algebra.

(2) $\Rightarrow$ (1): Suppose $\varphi=\prod \rho=\lambda_{1} \rho_{1}+\lambda_{2} \rho_{2}$, where $\lambda_{1}+\lambda_{2}=1, \lambda_{1}, \lambda_{2}>0$, and $\rho_{1}, \rho_{2} \in S_{\mathbb{B}_{\infty}}$. Let $A=D^{\otimes m}$. Similar to the proof in Theorem 14, we have $\rho_{1}=$ $\rho_{A} \otimes \rho_{A}=\rho^{\otimes 2 m}$ on $P F_{4 m}$. Let $m \rightarrow \infty$, we have $\rho_{1}=\prod \rho$. Therefore $\prod \rho$ is extremal.

Finally the equivalence of (3), (4), and (5) follows from Corollary 16.

Since every state in $S_{\mathbb{B}_{\infty}}$ can be written as a limit of the convex combination of the extremal states, such state is in the closure of the convex hull of the product states. This is the de Finetti theorem on parafermion algebra with square-free degree.

Theorem 18. Let d be squarefree. Then each state $\varphi \in S_{\mathbb{B}_{\infty}}$ on the $\mathbb{Z}_{d}$ graded parafermion algebra is neutral, and it can be expressed as the limit of convex combinations of product states $\prod \rho$, where $\rho \in S\left(P F_{2}\right)$ is neutral.

Corollary 19. If $\varphi^{\prime}=\frac{1}{2}\left(\prod \rho+\prod \tau\right)$ where $\rho, \tau \in S\left(P F_{2}\right)$ are distinct neutral states, then the von Neumann algebra $\overline{P F}_{\infty}^{\varphi^{\prime}}$ is not a factor.

\section{The de Finetti Theorem: The Non-neutral Case}

In this section, we consider the case when the square of some prime $p$ divides $d$, so $d=p^{2} d_{1}$. Let $p_{0}$ denote the smallest natural number such that $d \mid p_{0}^{2}$. Then $p_{0}<d$ and $p_{0} \mid d$. All the preliminary results in Sect. 4 hold in this case. The difference here is that when $d$ contains a square, the tail algebra may not be neutral. First, we consider the special case when the neutral part of the tail algebra is trivial.

Proposition 20. Let $\varphi \in S_{\mathbb{B}_{\infty}}$ and let $P F^{T, 0}=\mathbb{C}$, then there exists some $m_{0} \in \mathbb{N}$, with $p_{0}\left|m_{0}, m_{0}\right| d$, and such that

$$
P F^{T}=\bigoplus_{j=1}^{\frac{d}{m_{0}}} P F^{T, j m_{0}}, \quad \text { and where } \operatorname{dim}\left(P F^{T, j m_{0}}\right)=1 .
$$

Proof. We first show that $P F^{T}$ has a component with a charge $m>0$, only when $p_{0} \mid m$. Suppose that $p_{0}$ does not divide $m, x \in P F_{I}$, and $E_{T}(x) \in P F^{T, m}$. Then for some $k$ and some $J>I$, one has $\alpha^{k}(x) \in P F_{J}$. Using Proposition 11 and Proposition 5, we infer $E_{T}\left(x^{*} \alpha^{k}(x)\right)=E_{T}(x)^{*} E_{T}(x) \geqslant 0$ and $E_{T}\left(\alpha^{k}(x) x^{*}\right)=E_{T}(x) E_{T}(x)^{*} \geqslant 0$. Besides, from the canonical parafermion relations, $x^{*} \alpha^{k}(x)=q^{-m^{2}} \alpha^{k}(x) x^{*}$. Thus either $q^{-m^{2}}>0$ or else $E_{T}(x)=0$. The only positive value on the unit circle in the complex plane is 1 . But the condition $p_{0} \nmid m$ means $q^{-m^{2}} \neq 1$. So $E_{T}(x)=0$, and $\operatorname{dim}\left(P F^{T, m}\right)=0$. 
On the other hand suppose that $p_{0} \mid m$. We show that $\operatorname{dim}\left(P F^{T, m}\right) \leqslant 1$. In fact, for $A \in P F^{T, m}$, the quadratic expressions $A^{*} A$ and $A A^{*}$ are both neutral. Since we have assumed that $P F^{T, 0}=\mathbb{C}$, then $A^{*} A=\lambda \mathbb{I}$, and also $A A^{*}=\lambda^{\prime} I$, with $\lambda, \lambda^{\prime} \geqslant 0$, and equal to zero only if $A=0$. But then $\left(A^{*} A\right)^{2}=\lambda^{2} \mathbb{I}=\lambda \lambda^{\prime} \mathbb{I}$, so $\lambda=\lambda^{\prime}$. Therefore either $A=0$ or $U_{A}=A /\|A\|$ is unitary.

Thus for any two non-zero elements $A, B \in P F^{T, m}$, the neutral unitary $U_{A}^{*} U_{B}=e^{i \theta}$ is a phase, and $U_{B}=e^{i \theta} U_{A}$. In particular $\operatorname{dim}\left(P F^{T, m}\right) \leqslant 1$ as claimed. Thus

$$
P F^{T}=\bigoplus_{k=1}^{\frac{d}{p_{0}}} P F^{T, k p_{0}}, \quad \text { where } \operatorname{dim}\left(P F^{T, k p_{0}}\right) \leqslant 1 .
$$

Since the dimension of $P F^{T, k p_{0}}$ is either 0 or 1 , all $k p_{0}$ such that $\operatorname{dim}\left(P F^{T, k p_{0}}\right)=1$ form a subgroup of $\mathbb{Z}_{d}$. There exists a smallest such number $k_{0}$ that divides all $\mathrm{k}$ with $\operatorname{dim}\left(P F^{T, k p_{0}}\right)=1$. Hence $P F^{T}$ can be written as

$$
P F^{T}=\bigoplus_{j=1}^{\frac{d}{m_{0}}} P F^{T, j m_{0}}, \quad \text { with } \operatorname{dim} P F^{T, j m_{0}}=1,
$$

where $m_{0}=k_{0} p_{0}$.

Define $P F_{2}^{p_{0} \mathbb{Z}}=\left\{x \in P F_{2} \mid \operatorname{deg}(x) \in p_{0} \mathbb{Z} / d \mathbb{Z}\right\}$. Then for any $x, y \in P F_{2}^{p_{0} \mathbb{Z}}$,

$$
\alpha^{j}(x) \alpha^{k}(y)=q^{\operatorname{deg}(x) \operatorname{deg}(y)} \alpha^{k}(y) \alpha^{j}(x)=\alpha^{k}(y) \alpha^{j}(x), \forall j \neq k .
$$

Therefore for any state $\rho$ on $P F_{2}$ with density matrix $D$ in $P F_{2}^{p_{0} \mathbb{Z}}$, the product state $\prod \rho$ is a well-defined state on $P F_{\infty}$.

Theorem 21. Let $\varphi \in S_{\mathbb{B}_{\infty}}$. Then the following statement are equivalent:

$\left(1^{\prime}\right) \varphi$ is extremal in $S_{\mathbb{B}_{\infty}}$.

$\left(2^{\prime}\right) \varphi=\prod \rho$, where $\rho$ is a state on $P F_{2}$ with a density matrix in $P F_{2}^{p_{0} \mathbb{Z}}$.

Proof. $\left(2^{\prime}\right) \Rightarrow\left(1^{\prime}\right)$ : The proof is the same as the proof of $(2) \Rightarrow(1)$ in Theorem 17.

$\left(1^{\prime}\right) \Rightarrow\left(2^{\prime}\right)$ : If $\varphi$ is extremal, then Proposition 12 shows that $P F^{T, 0}=\mathbb{C}$. Using Proposition 20, we infer (65), and that $P F^{T, j m_{0}}$ is generated by a unitary $U_{j}$ with charge $j$. We can choose $\left\{U_{j}\right\}$, so that the set $G=\left\{U_{j}\right\}$ is a cyclic group. The restriction $\varphi_{G}$ of the state $\varphi$ to $G$ is a convex linear combination of characters $\chi_{j}$ on $G$, namely $\varphi_{G}=\sum_{j} \lambda_{j} \chi_{j}$, where $\lambda_{j} \geqslant 0$ and $\sum_{j} \lambda_{j}=1$.

Each character $\chi_{i}$ can be extended to a state on $P F^{T}$, the group algebra of $G$. It can also be extended to a state on the parafermion algebra,

$$
\chi_{i}(x):=\chi_{i}\left(E_{T}(x)\right), \forall x \in \overline{P F}_{\infty}^{\varphi}
$$

as $E_{T}$ is a conditional expectation. Then $\chi_{i}(x)=0$, for any element $x \in \overline{P F}_{\infty}^{\varphi}$ orthogonal to $P F^{T}$. By Proposition $9, P F^{T}$ is $\mathbb{B}_{\infty}$ invariant, so $\chi_{i}$ is $\mathbb{B}_{\infty}$ invariant. Since $\varphi$ is extremal, $\varphi_{G}$ is equal to some character $\chi=\chi_{i}$, otherwise the state $\varphi$ can be written as the convex combination of the $\mathbb{B}_{\infty}$-invariant states. Since $\chi$ is a character, thus

$$
\chi\left(E_{T}(x) E_{T}(y)\right)=\chi\left(E_{T}(x)\right) \chi\left(E_{T}(y)\right) .
$$


That is, $\varphi\left(E_{T}(x) E_{T}(y)\right)=\varphi\left(E_{T}(x)\right) \varphi\left(E_{T}(y)\right)$. By Proposition $11, \varphi=\prod \rho$ for some state $\rho$ on $P F_{2}$.

Let $D=\sum_{k \in \mathbb{Z}_{d}} D_{k}$ be the density matrix of $\rho$, and $D_{k} \neq 0$ in $P F_{2}^{k}$. Then $\varphi\left(D_{k}^{*} \alpha\left(D_{k}^{*}\right)\right)=\rho\left(D_{k}^{*}\right)^{2}>0$. On the other hand

$$
\varphi\left(D_{k}^{*} \alpha\left(D_{k}^{*}\right)\right)=q^{k^{2}} \varphi\left(\alpha\left(D_{k}^{*}\right) D_{k}^{*}\right)=q^{k^{2}} \rho\left(D_{k}^{*}\right)^{2} .
$$

So $q^{k^{2}}=1$ and $p_{0} \mid k$. Therefore $D \in P F_{2}^{p_{0} \mathbb{Z}}$.

Theorem 22. Given a state $\varphi \in S_{\mathbb{B}_{\infty}}$, then the following statements are equivalent:

(3') $P F^{T, 0}=\mathbb{C}$.

(4') $\overline{P F}_{\infty}^{\varphi}$ is a factor.

${ }^{\left(5^{\prime}\right)} \varphi=\sum_{j} \lambda_{j} \chi_{i} \circ \prod \rho$, namely for any homogenous $x \in \overline{P F}^{\varphi}$,

$$
\varphi(x)=\sum_{j} \lambda_{j} \chi_{i}(\operatorname{deg}(x))\left(\prod \rho\right)(x),
$$

and the density matrix of $\rho$ is in $P F_{2}^{p_{0} \mathbb{Z}}$.

Proof. Proposition 10 ensures the equivalence between $\left(3^{\prime}\right)$ and $\left(4^{\prime}\right)$.

$\left(3^{\prime}\right) \Rightarrow\left(5^{\prime}\right)$ : If $P F^{T, 0}=\mathbb{C} 1$, then based on the proof of $\left(1^{\prime}\right) \Rightarrow\left(2^{\prime}\right)$, the state restricted in the cyclic group $\mathrm{G} \varphi_{G}$ can be expressed as the convex combination of characters $\sum_{i} \lambda_{i} \chi_{i}$. For any $A \in P_{I}$ and $B \in P_{J}, I<J$. We only need to consider the case where $m_{0}\left|\operatorname{deg}(A), m_{0}\right| \operatorname{deg}(B)$. Thus

$$
\begin{aligned}
\varphi(A B) & =\varphi\left(E_{T}(A B)\right) \\
& =\sum_{i} \lambda_{i} \chi_{i}\left(E_{T}(A B)\right) \\
& =\sum_{i} \lambda_{i} \chi_{i}(\operatorname{deg}(A) \operatorname{deg}(B)) \chi_{0}\left(E_{T}(A B)\right) .
\end{aligned}
$$

Due to the proof of $\left(1^{\prime}\right) \Rightarrow\left(2^{\prime}\right)$, each irreducible character corresponds to one state which can be written as the product state. Thus, the state $\chi_{0} \circ E_{T}$ can be written as $\prod \rho$, and the density matrix of $\rho$ is in $P F_{2}^{p_{0} \mathbb{Z}}$. Therefore $\varphi=\sum_{i} \lambda_{i} \chi_{i} \circ \prod \rho$.

$\left(5^{\prime}\right) \Rightarrow\left(3^{\prime}\right)$ : Let $\left\{b_{k}\right\}_{k \in \mathbb{Z}_{d}}$ be an orthonormal basis of $P F_{2}$ with inner product $\langle x, y\rangle=$ $\varphi\left(y^{*} x\right)$, such that $b_{0}=I$. Since $\varphi$ can be written as $\sum_{i} \lambda_{i} \chi_{i} \circ \prod \rho$, then any element $x \in P F^{T, 0}$ has the orthogonal decomposition

$$
x=\beta_{0} I+\sum_{i=1}^{\infty} x_{i},
$$

where

$$
x_{i}=\sum_{k=1}^{d-1} \beta_{i, k} \alpha^{i-1}\left(b_{k}\right) \alpha^{i}\left(x_{i, k}\right),
$$

for some $\beta_{i, k} \in \mathbb{C}, x_{i, k} \in \overline{P F}^{\varphi}$, such that $\operatorname{deg}\left(x_{i}\right)=0$ and $\varphi\left(x_{j}^{*} x_{i}\right)=\prod \rho\left(x_{j}^{*} x_{i}\right)=0$, for $i \neq j$. Besides, $\alpha(x)=x$, which implies that $x_{i}=0$ for $i \geqslant 1$. That is, all the element in $P F^{T, 0}$ is in proportion to the identity. Thus, we obtain $\left(5^{\prime}\right)$. 
Besides, the condition $\left(2^{\prime}\right)$ can always implies the condition $\left(5^{\prime}\right)$, these five conditions can be summarized as follows.

$$
\begin{gathered}
\left(1^{\prime}\right) \Leftrightarrow\left(2^{\prime}\right) \\
\Downarrow \\
\left(3^{\prime}\right) \Leftrightarrow\left(4^{\prime}\right) \Leftrightarrow\left(5^{\prime}\right)
\end{gathered}
$$

That is, for the extremal $\mathbb{B}_{\infty}$-invariant state, the corresponding tail algebra $P F^{T}$ can be decomposed as (65).

\section{Summary}

We have proposed and proved a new type of de Finetti theorem for the parafermion algebra $P F_{\infty}$ with respect to the action of braid group $\mathbb{B}_{\infty}$ that braids qudits (pairs of parafermions). We have two results based on whether or not the degree $d$ of the given parafermion algebra is square free. In both cases, we characterize the extremal, braidinvariant states, and show that these properties are equivalent to the states being product states on $\prod P F_{2} \cong \prod \mathbb{M}_{d}$.

In the square-free case, we have found other equivalent conditions for a braid-invariant state $\varphi$ to be extremal. It is surprising that one such condition is that the von Neumann algebra $\overline{P F}_{\infty}^{\varphi}$ is a factor; so if $\varphi$ is the convex combination of extremal, braid-invariant states, then the corresponding von Neumann algebra of parafermions is not a factor. Since the parafermion algebra has been used to establish a framework of topological quantum information theory, the de Finetti theorem in this work can shed insight on the topological quantum information theory.

Acknowledgement. All the authors thank Harvard University for hospitality during the conception and writing of this paper. We are grateful to Claus Köstler, as well as to the anonymous referee, for a number of helpful comments on earlier versions of our paper. We thank the Templeton Religion Trust for support of this research under Grants TRT0080 and TRT0159. Kaifeng Bu was partially supported with an Academic Award for Outstanding Doctoral Candidates from Zhejiang University. Arthur Jaffe was partially supported by ARO Grant W911NF1910302. Jinsong Wu was supported by NSFC 11771413.

Open Access This article is distributed under the terms of the Creative Commons Attribution 4.0 International License (http://creativecommons.org/licenses/by/4.0/), which permits unrestricted use, distribution, and reproduction in any medium, provided you give appropriate credit to the original author(s) and the source, provide a link to the Creative Commons license, and indicate if changes were made.

Publisher's Note Springer Nature remains neutral with regard to jurisdictional claims in published maps and institutional affiliations.

\section{References}

[BCS12] Banica, T., Curran, S., Speicher, R.: De Finetti theorems for easy quantum groups. Ann. Probab. 40(1), 401-435 (2012)

[BL09] Barrett, J., Leifer, M.: The de Finetti theorem for test spaces. New J. Phys. 11(3), 033024 (2009)

[BrCY11] Brandão, F.G.S.L., Christandl, M., Yard, J.: A Quasipolynomial-time algorithm for the quantum separability problem. In: STOC 11 Proceedings of the 43rd ACM Symposium on Theory of Computing, pp. 343-352 (2011). ACM

[BrP10] Brandão, F.G.S.L., Plenio, M.B.: A generalization of quantum Stein's lemma. Commun. Math. Phys. 295(3), 791-828 (2010)

[BrH17] Brandão, F.G.S.L., Harrow, A.W.: Quantum de Finetti theorems under local measurements with applications. Commun. Math. Phys. 353(2), 469-506 (2017) 
[CAS13] Clarke, D.J., Alicea, J., Shtengel, K.: Exotic non-abelian anyons from conventional fractional quantum Hall states. Nat. Commun. 4, 1348 (2013)

[CFS02] Caves, C.M., Fuchs, C.A., Schack, R.: Unknown quantum states: the quantum de Finetti representation. J. Math. Phys. 43(9), 4537 (2002)

[CF12] Crismale, V., Fidaleo, F.: De Finetti theorem on the CAR algebra. Commun. Math. Phys. 315(1), 135-152 (2012)

[CK07] Christandl, M., König, R., Mitchison, G., Renner, R.: One-and-a-half quantum de Finetti theorems. Commun. Math. Phys. 273(2), 473-498 (2007)

[CT09] Christandl, M., Toner, B.: Finite de Finetti theorem for conditional probability distributions describing physical theories. J. Math. Phys. 50, 042104 (2009)

[Cur09] Curran, S.: Quantum exchangeable sequences of algebras. Indiana Univ. Math. J. 58, 1097-1126 (2009)

[Cur10] Curran, S.: Quantum rotatability. Trans. Am. Math. Soc. 362, 4831-4851 (2010)

[DK14] Dykema, K., Köstler, C.: Tail algebras of quantum exchangeable random variables. Proc. AMS 142(11), 3853-3863 (2014)

[dF31] de Finetti, B.: Funzione caratteristica di un fenomeno aleatorio. Atti della R. Accademia Nazionale dei Lincei, Ser. 6, Memorie, Classe di Scienze Fisiche, Matematiche e Naturali 4, 251-299(1931)

[dF37] de Finetti, B.: La prévision: ses lois logiques, ses sources subjectives. Ann. Inst. H. Poincaré 7, 1-68 (1937)

[DF80] Diaconis, P., Freedman, D.: Finite exchangeable sequences. Ann. Probab. 8(4), 745-764 (1980)

[Fen12] Fendley, P.: Parafermionic edge zero modes in $Z_{n}$-invariant spin chains. J. Stat. Mech. 2012(11), P11020 (2012)

[FLV88] Fannes, M., Lewis, J.T., Verbeure, A.: Symmetric states of composite systems. Lett. Math. Phys. 15(3), 255-260 (1988)

[GK09] Gohm, R., Köstler, C.: Noncommutative independence from the braid group $\mathbb{B}_{\infty}$. Commun. Math. Phys. 289(2), 435-482 (2009)

[GK10] Gohm, R., Köstler, C., Gohm, R., Köstler, C.: Noncommutative Independence from Characters of the Infinite Symmetric Group $\mathbb{S}_{\infty}$. arXiv: 1005.5726

[EL55] Hewitt, E., Leonard, J.S.: Symmetric measures on cartesian products. Trans. Am. Math. Soc. 80, 470-501 (1955)

[HHHH09] Horodecki, R., Horodecki, P., Horodecki, M., Horodecki, K.: Quantum entanglement. Rev. Mod. Phys. 81, 865-942 (2009)

[HM] Hudson, R.L., Moody, G.R.: Locally normal symmetric states and an analogue of de Finetti's theorem. Probab. Theory Rel. 33, 343-351 (1976)

[HWL15] Hutter, A., Wootton, J.R., Loss, D.: Parafermions in a Kagome lattice of qubits for topological quantum computation. Phys. Rev. X 5, 041040 (2015)

[JL17] Jaffe, A., Liu, Z.: Planar para algebras, reflection positivity. Commun. Math. Phys. 352(1), 95-133 (2017)

[JLW17] Jaffe, A., Liu, Z., Wozniakowski, A.: Constructive simulation and topological design of protocols. New J. Phys. 19(6), 063016 (2017)

[JLW18] Jaffe, A., Liu, Z., Wozniakowski, A.: Holographic software for quantum networks. Sci. China Math. 61(4), 593-626 (2018)

[Kit03] Kitaev, A.Y.: Fault-tolerant quantum computation by anyons. Ann. Phys. 303(1), 2-30 (2003)

[KL14] Klinovaja, J., Loss, D.: Parafermions in an interacting nanowire bundle. Phys. Rev. Lett. 112, 246403 (2014)

[KR05] König, R., Renner, R.: A de Finetti representation for finite symmetric quantum states. J. Math. Phys. 46(12), 122108 (2005)

[K10] Köstler, C.: A noncommutative extended de Finetti theorem. J. Funct. Anal. 258(4), 1073-1120 (2010)

[KS09] Köstler, C., Speicher, R.: A noncommutative de Finetti theorem: invariance under quantum permutations is equivalent to freeness with amalgamation. Comm. Math. Phys. 291(2), 473-490 (2009)

[LBRS12] Lindner, N.H., Berg, E., Refael, G., Stern, A.: Fractionalizing majorana fermions: non-abelian statistics on the edges of abelian quantum Hall states. Phys. Rev. X 2, 041002 (2012)

[Liu15] Liu, W.: A noncommutative de Finetti theorem for boolean independence. J. Funct. Anal. 269(7), 1950-1994 (2015)

[Liu17] Liu, W.: Extended de Finetti theorems for boolean independence and monotone independence. Trans. Am. Math. Soc. 370, 1959-2003 (2018)

[LS15] Li, K., Smith, G.: Quantum de Finetti theorem under fully-one-way adaptive measurements. Phys. Rev. Lett. 114, 160503 (2015)

[LWJ17] Liu, Z., Wozniakowski, A., Jaffe, A.: Quon 3d language for quantum information. Proc. Natl. Acad. Sci. 114, 2497-2502 (2017) 
[MCA+14] Mong, R.S.K., Clarke, D.J., Alicea, J., Lindner, N.H., Fendley, P., Nayak, C., Oreg, Y., Stern, A., Berg, E., Shtengel, K., Fisher, M.P.A.: Universal topological quantum computation from a superconductor-abelian quantum Hall heterostructure. Phys. Rev. X 4, 011036 (2014)

[NSS+08] Nayak, C., Simon, S.H., Stern, A., Freedman, M., Sarma, S.D.: Non-abelian anyons and topological quantum computation. Rev. Mod. Phys. 80, 1083-1159 (2008)

[Ren05] Renner, R.: Security of quantum key distribution, arXiv:quant-ph/0512258

[Ren07] Renner, R.: Symmetry of large physical systems implies independence of subsystems. Nat. Phys. 3, 645-649 (2007)

[RW89] Raggio, G.A., Werner, R.F.: Quantum statistical mechanics of general mean field systems. Helv. Phys. Acta 62, 980-1003 (1989)

[RS55] Riesz, F., Sz.-Nagy, B.: Functional Analysis. Frederick Ungar Publishing Company, New York (1955)

[Sto69] Størmer, E.: Symmetric states of infinite tensor products of $C^{*}$-algebras. J. Funct. Anal. 3(1), 48-68 (1969)

[YW12] You, Y.-Z., Wen, X.-G.: Projective non-abelian statistics of dislocation defects in a $\mathbb{Z}_{N}$ rotor model. Phys. Rev. B 86, 161107 (2012)

Communicated by M. M. Wolf 